\title{
Robustness Analysis of a Type of Iterative Algorithm for R-L Fractional Nonlinear Control Systems in the Sense of $L_{p}$ Norm
}

\author{
Yanfang Li, Xianghu Liu (D), and Guangjun Xu \\ Department of Mathematics, Zunyi Normal College, Zunyi 563006, Guizhou, China \\ Correspondence should be addressed to Xianghu Liu; liouxianghu04@126.com
}

Received 28 October 2020; Revised 4 December 2020; Accepted 31 December 2020; Published 16 January 2021

Academic Editor: Abdelalim Elsadany

Copyright (c) 2021 Yanfang Li et al. This is an open access article distributed under the Creative Commons Attribution License, which permits unrestricted use, distribution, and reproduction in any medium, provided the original work is properly cited.

The paper is concerned with the robustness analysis of a type of iterative algorithm for R-L fractional nonlinear control systems in the sense of $L_{p}$ norm. Firstly, according to the Laplace transform and M-L function, the concept of mild solutions of the system is derived. Secondly, we give the sufficient conditions of robustness analysis of the $P D^{\alpha}$-type ILC algorithm with uncertain disturbances and then study the robust analysis of the second-order $P D^{\alpha}$-type ILC algorithm. At last, two fractional examples are given to demonstrate the results.

\section{Introduction}

The aim of the paper is to analyze the robustness of a type of iterative algorithm in the sense of $L_{p}$ norm of the following R-L fractional system:

$$
\left\{{ }^{\mathrm{RL}} D_{t}^{\alpha} z(t)=A z(t)+B u(t), \quad t \in J=[0, b],\left(g_{1-\alpha} * z\right)(0)=z_{0}, y(t)=C z(t)+D u(t),\right.
$$

where ${ }^{\mathrm{RL}} D_{t}^{\alpha}$ denotes the $\mathrm{R}-\mathrm{L}$ derivative of order $\alpha, 0<\alpha<1$, $A, B, C \in R^{n \times n}, \quad u(t)$ is a control vector, and $g_{1-\alpha}=\left(t^{1-\alpha} / \Gamma(1-\alpha)\right)$.

Iterative learning control (ILC) was shown by Uchiyama in 1978 (in Japanese), and in recent years, more and more scholars have paid attention to the problems, among which are experts who study fractional calculus. The work of the fractional-order system in iterative learning control appeared in 2001. In the following decade, extensive attention has been paid to this field, great progress has been made [1-8], and many fractional nonlinear systems were investigated [9-17]. In recent years, the fractional ILC algorithm has played a great role in multiagent control information transmission, and for more information, one can see the references [13-16].
In Li et al.'s study [17], the authors discussed a P-type ILC scheme for a class of fractional-order nonlinear systems with delay by using the $\lambda$-norm and Gronwall inequality and obtained the sufficient condition for the robust convergence of the tracking errors.

In view of that the $\lambda$-norm often causes tracking errors that exceed the actual engineering range and cause inaccurate data, the authors Lan and Lin [18] used the $L_{p}$ norm to discuss the convergence of iterative learning algorithms, and it objectively quantifies the essential characteristics of the tracking error and comprehensively reflects the behavior of the system. Zhang and Peng [19] used the generalized Young inequality of convolution and discussed the robustness of the PD-type fractional-order iteration and learning control algorithm in the sense of $L_{p}$ norm, and the conditions of its robust convergence are obtained. 
The above references have analyzed the robustness of the algorithm of the Caputo-type fractional system, and we find the Caputo fractional derivative is often used to solve general diffusion problems. The R-L type fractional derivative has a wider application in viscoelastic problems because it does not require the function to be differentiable at the origin. As far as we all know, analyzing robustness with interference of the R-L type fractional system is an extremely interesting and challenging work.

The rest of this paper is organized as follows. In Section 2, according to the Laplace transform and M-L function, the concept of mild solutions of the system is derived. In Section 3 , we give the sufficient conditions of robustness analysis of the $P D^{\alpha}$-type ILC algorithm with uncertain disturbances and then study the robust analysis of the second-order $P D^{\alpha}$-type ILC algorithm. In Section 4, two fractional examples are given to demonstrate the results.

\section{Some Preliminaries for Fractional Systems}

In this section, we show some definitions and preliminaries of the $L_{p}$ norm and Mittag-Leffler function. From [20-23], one can see the definitions of the R-L fractional integral and derivative.

Definition 1. The norm for the $n$-dimensional vector $Z=$ $\left(z_{1}, z_{2}, \ldots, z_{n}\right)$ is defined as $\|Z\|_{T}=\max _{1 \leq i \leq n}\left|z_{i}\right|$, and the $L_{p}$ norm is defined as $|Z|_{p}=\left[\int_{0}^{T}\left(\max \left|z_{i}\right|\right)^{p} \mathrm{~d} t\right]^{(1 / p)}$, where $t \in[0, T]$.

Definition 2. The definition of the two-parameter function of the Mittag-Leffler type is described by

$$
E_{\alpha, \beta}(z)=\sum_{k=0}^{\infty} \frac{z^{k}}{\Gamma(\alpha k+\beta)}, \quad \alpha>0, \beta>0, z \in C .
$$

$$
\left\{{ }^{\mathrm{RL}} D_{t}^{\alpha} z_{k}(t)=A z_{k}(t)+B u_{k}(t)+\omega(t)\right.
$$

where $k=0,1,2,3, \ldots$, and $\omega(t)$ and $\nu(t)$ are uncertain disturbances.

For system (6), we apply the following open- and closedloop $P D^{\alpha}$-type ILC algorithm:

$$
u_{k+1}(t)=u_{k}(t)+\gamma_{1} e_{k}(t)+\gamma_{2} e_{k+1}^{(\alpha)}(t),
$$

where $t \in[0, b], \gamma_{1}$ and $\gamma_{2}$ are the parameters which will be determined, $y_{d}(t)$ is the given function, $e_{k}=y_{d}(t)-y_{k}(t)$, and $e_{k}^{(\alpha)}(t)={ }^{\mathrm{RL}} D_{t}^{\alpha} e_{k}$. For convenience, one can see Figure 1. The initial state of each iterative learning is as follows:

$$
z_{k+1}(0)=z_{k}(0)+B \gamma_{1} e_{k}(t)
$$

We denote that
If $\beta=1$, one has the Mittag-Leffler function of one parameter as follows:

$$
E_{\alpha}(z)=\sum_{k=0}^{\infty} \frac{z^{k}}{\Gamma(\alpha k+1)}
$$

Now, according to the results of the papers [17, 24-27], we will give the following lemma.

Lemma 1 (Lemma 3, see [25]). The general solution of equation (1) is given by

$$
z(t)=t^{\alpha-1} E_{\alpha, \alpha}(A, t) z_{0}+\int_{0}^{t}(t-s)^{\alpha-1} E\left(A(t-s)^{\alpha}\right) B u(s) \mathrm{d} s
$$

where

$$
E_{\alpha, \beta}(A, t)=\sum_{k=0}^{\infty} \frac{A^{k} t^{\alpha k+\beta-1}}{\Gamma(\alpha k+\beta)}
$$

Lemma 2 (Definition 2.4, see [27]). The operators $E_{\alpha, \alpha}(t)$ are exponentially bounded, and there is a constant $C_{0}=(1 / \alpha)\|A\|^{((1-\alpha) / \alpha)}, \quad e_{\alpha}(t)=e^{\|A\|^{(1 / \alpha)} t}, \quad M=e_{\alpha}(b)$, and $\left\|E_{\alpha, \alpha}(A, t)\right\| \leq C_{0} e_{\alpha}(t) \leq C_{0} M$.

Lemma 3 (Hölder inequality). Set $p>0, q>0$, and $(1 / p)+(1 / q)=1$; $\quad$ if $\quad f \in L^{p}(\Omega), g \in L^{q}(\Omega), \quad$ and $f \cdot g \in L^{1}(\Omega)$, then $\|f \cdot g\|_{L^{1}} \leq\|f\|_{L^{p}}\|g\|_{L^{q}}$.

\section{Robustness Analysis of the $P D^{\alpha}$-Type ILC Algorithm with Uncertain Disturbances}

In this section, we consider the following fractional equation: $t \in J=[0, b], y_{k}(t)=C z_{k}(t)+D u_{k}(t)+v(t)$,

$$
\begin{gathered}
\kappa_{1}=\left\|I+\gamma_{2} D\right\|-\frac{b^{\alpha-(1 / p)}\left\|\gamma_{2} C\right\| C_{0} M\|B\|}{\sqrt[q]{q(\alpha-1)+1}}, \\
\kappa_{2}=\left\|I-\gamma_{1} D\right\|-\frac{b^{\alpha-(1 / p)}\left\|\gamma_{1} C\right\| C_{0} M\|B\|}{\sqrt[q]{q(\alpha-1)+1}}, \\
\kappa_{3}=\frac{b^{\alpha-(1 / p)}\left(\left\|\gamma_{1} C\right\|+\left\|\gamma_{2} C\right\|\right) C_{0} M\|B\|\|\omega\|_{L_{p}}}{\sqrt[q]{q(\alpha-1)+1}} \\
+\left(\left\|\gamma_{1}\right\|+\left\|\gamma_{2}\right\|\right)\|v\|_{L_{p}} .
\end{gathered}
$$


Theorem 1. Assume that each iteration state meets algorithm (7) and the initial state is $z_{k}(0)=z_{d}(0)$; then, there exists $m>0$ such that $\kappa_{1}>0, \kappa_{2}>0, \kappa_{3}<m$, and $\kappa_{1}>\kappa_{2}$, and then, the sufficient condition for being uniformly bounded on $J$ is $\lim _{k \rightarrow \infty}\left\|u_{k}\right\|_{L_{p}} \leq\left(\kappa_{3} /\left(\kappa_{1}-\kappa_{2}\right)\right)$.

Proof. Define

$$
\left\{\begin{array}{l}
\Delta z_{k}(t)=z_{d}(t)-z_{k}(t), \\
\Delta u_{k}(t)=u_{d}(t)-u_{k}(t) .
\end{array}\right.
$$

For $t \in J$, one has $\Delta z_{k}^{(\alpha)}(t)={ }^{\mathrm{RL}} D_{t}^{\alpha} \Delta z_{k}(t)=A \Delta z_{k}(t)+$ $B \Delta u_{k}(t)$ and $e_{k+1}^{(\alpha)}(t)=C\left(A \Delta z_{k+1}(t)+B \Delta u_{k+1}(t)\right)$.

According to system (6), we have

$$
\begin{aligned}
z_{k+1}(t)= & t^{\alpha-1} E_{\alpha, \alpha}(A, t) z_{0}+ \\
& \cdot \int_{0}^{t}(t-s)^{\alpha-1} E_{\alpha, \alpha}\left(A(t-s)^{\alpha}\right)\left(B u_{k+1}(s)+\omega(t)\right) \mathrm{d} s, \\
z_{d}(t)= & t^{\alpha-1} E_{\alpha, \alpha}(A, t) z_{0}+ \\
& \cdot \int_{0}^{t}(t-s)^{\alpha-1} E_{\alpha, \alpha}\left(A(t-s)^{\alpha}\right) B u_{d}(s) \mathrm{d} s,
\end{aligned}
$$

and thus, using the ILC algorithms (7) and (8), we derive

$$
\begin{aligned}
\Delta z_{k+1}(t)= & \int_{0}^{t}(t-s)^{\alpha-1} E_{\alpha, \alpha}\left(A(t-s)^{\alpha}\right) B \\
& \cdot\left(\Delta u_{k+1}(s)+\omega(t)\right) \mathrm{d} s, \\
\Delta z_{k}(t)= & \int_{0}^{t}(t-s)^{\alpha-1} E_{\alpha, \alpha}\left(A(t-s)^{\alpha}\right) B \\
& \cdot\left(\Delta u_{k}(s)+\omega(t)\right) \mathrm{d} s,
\end{aligned}
$$

$$
\begin{aligned}
\Delta u_{k+1}(t)= & \Delta u_{k}(t)-\gamma_{1}\left(y_{d}(t)-y_{k}(t)\right)-\gamma_{2}\left(y_{d}(t)-y_{k+1}(t)\right) \\
= & \Delta u_{k}(t)-\gamma_{1}\left(C \Delta z_{k}(t)+D \Delta u_{k}(t)-v(t)\right)-\gamma_{2}\left(C \Delta z_{k+1}(t)+D \Delta u_{k+1}(t)-v(t)\right) \\
= & \Delta u_{k}(t)-\left(\gamma_{1} C \int_{0}^{t}(t-s)^{\alpha-1} E_{\alpha, \alpha}\left(A(t-s)^{\alpha}\right) B\left(\Delta u_{k}(s)+\omega(t)\right) \mathrm{d} s+\gamma_{1} D \Delta u_{k}(t)-\gamma_{1} \nu(t)\right) \\
& -\left(\gamma_{2} C \int_{0}^{t}(t-s)^{\alpha-1} E_{\alpha, \alpha}\left(A(t-s)^{\alpha}\right) B\left(\Delta u_{k+1}(s)+\omega(t)\right) \mathrm{d} s+\gamma_{2} D \Delta u_{k+1}(t)-\gamma_{2} \nu(t)\right)
\end{aligned}
$$

Hence,

$$
\begin{aligned}
& \Delta u_{k+1}(t)\left(I+\gamma_{2} D\right)=\Delta u_{k}(t)\left(I-\gamma_{1} D\right) \\
& \quad-\left(\gamma _ { 1 } C \int _ { 0 } ^ { t } ( t - s ) ^ { \alpha - 1 } E _ { \alpha , \alpha } ( A ( t - s ) ^ { \alpha } ) B \left(\Delta u_{k}(s)\right.\right. \\
& \left.\quad+\omega(t)) \mathrm{d} s-\gamma_{1} v(t)\right) \\
& \quad-\left(\gamma _ { 2 } C \int _ { 0 } ^ { t } ( t - s ) ^ { \alpha - 1 } E _ { \alpha , \alpha } ( A ( t - s ) ^ { \alpha } ) B \left(\Delta u_{k+1}(s)\right.\right. \\
& \left.+\omega(t)) \mathrm{d} s-\gamma_{2} v(t)\right) .
\end{aligned}
$$

By taking the $L_{p}$ norm, we obtain

$$
\begin{aligned}
& \left\|\Delta u_{k+1}\right\|_{L^{p}}\left\|\left(I+\gamma_{2} D\right)\right\| \leq\left\|\Delta u_{k}\right\|_{L^{p}}\left\|\left(I+\gamma_{2} D\right)\right\| \\
& +\frac{b^{\alpha-(1 / p)}\left\|\gamma_{1} C\right\| C_{0} M\left(\|B\|\left\|\Delta u_{k}\right\|_{L^{p}}+\|\Delta \omega\|_{L^{p}}\right)}{\sqrt[q]{q(\alpha-1)+1}}+\left\|\gamma_{1}\right\|\|\nu\|_{L^{p}} \\
& +\frac{b^{\alpha-(1 / p)}\left\|\gamma_{2} C\right\| C_{0} M\left(\|B\|\left\|\Delta u_{k+1}\right\|_{L^{p}}+\|\Delta \omega\|_{L^{p}}\right)}{\sqrt[q]{q(\alpha-1)+1}}+\left\|\gamma_{2}\right\|\|\nu\|_{L^{p}},
\end{aligned}
$$

denoting

$$
\begin{aligned}
\kappa_{1}=\left\|I+\gamma_{2} D\right\|-\frac{b^{\alpha-(1 / p)}\left\|\gamma_{2} C\right\| C_{0} M\|B\|}{\sqrt[q]{q(\alpha-1)+1}}, \\
\kappa_{2}=\left\|I-\gamma_{1} D\right\|-\frac{b^{\alpha-(1 / p)}\left\|\gamma_{1} C\right\| C_{0} M\|B\|}{\sqrt[q]{q(\alpha-1)+1}}, \\
\kappa_{3}=\frac{b^{\alpha-(1 / p)}\left(\left\|\gamma_{1} C\right\|+\left\|\gamma_{2} C\right\|\right) C_{0} M\|B\|\|\omega\|_{L_{p}}}{\sqrt[q]{q(\alpha-1)+1}} \\
+\left(\left\|\gamma_{1}\right\|+\left\|\gamma_{2}\right\|\right)\|\nu\|_{L_{p} .}
\end{aligned}
$$

Consequently, $\kappa_{1}\left\|\Delta u_{k+1}\right\|_{L^{p}} \leq \kappa_{2}\left\|\Delta u_{k}\right\|_{L^{p}}+\kappa_{3}$. So, there exists a positive $m$, such that $\kappa_{3}<m$ and $\kappa_{1}>0, \kappa_{2}>0$, and $\kappa_{1}>\kappa_{2}$, and then $\lim _{k \rightarrow \infty}\left\|u_{k}\right\|_{L_{p}} \leq\left(\kappa_{3} /\left(\kappa_{1}-\kappa_{2}\right)\right)$, which implies $e_{k}(t)$ is uniformly bounded on $J$. 


\section{Robust Analysis of the Second-Order $P D^{\alpha}$-Type ILC Algorithm}

In this section, we consider the following second-order $P D^{\alpha}$-type ILC algorithm:

$$
\begin{aligned}
u_{2}(t)= & u_{1}(t)+\gamma_{1} e_{1}(t)+\gamma_{2} e_{1}^{(\alpha)}(t), \\
u_{k+1}(t)= & r_{1}\left[u_{k}(t)+\gamma_{1} e_{k}(t)+\gamma_{2} e_{k}^{(\alpha)}(t)\right] \\
& +r_{2}\left[u_{k-1}(t)+\gamma_{3} e_{k-1}(t)+\gamma_{4} e_{k-1}^{(\alpha)}(t)\right], \quad k=2,3, \ldots,
\end{aligned}
$$

where $r_{1}+r_{2}=1$.

The initial state of the system is as follows:

$$
z_{k+1}(0)=z_{k}(0)+B L_{1} e_{k}(t)+B L_{2} e_{k}^{(\alpha)}(t) .
$$

For convenience, one can see Figure 2.

Assume that the initial state of each iterative learning meets (18), where $L_{1}$ and $L_{2}$ are the parameters which will be determined.

Note

$$
\begin{aligned}
K_{1}= & \left\|r_{1}+r_{1} \gamma_{1} D+r_{1} \gamma_{2} C B\right\| \\
& +\left\|r_{1} \gamma_{1} C+r_{1} \gamma_{2} C A\right\| \frac{b^{\alpha-(1 / p)} C_{0} M\|B\|}{\sqrt[q]{q(\alpha-1)+1}}, \\
K_{2}= & \left\|r_{2}+r_{2} \gamma_{3} D+r_{2} \gamma_{4} C B\right\| \\
& +\left\|r_{2} \gamma_{3} C+r_{2} \gamma_{4} C A\right\| \frac{b^{\alpha-(1 / p)} C_{0} M\|B\|}{\sqrt[q]{q(\alpha-1)+1}}, \\
K_{3}= & \left(\left\|r_{1} \gamma_{1} C+r_{1} \gamma_{2} C A\right\|\right. \\
& \left.+\left\|r_{2} \gamma_{3} C+r_{2} \gamma_{4} C A\right\|\right) \frac{b^{\alpha-(1 / p)} C_{0} M}{\sqrt[q]{q(\alpha-1)+1}}\|\omega\|_{L_{p}} .
\end{aligned}
$$

Theorem 2. Suppose system (6) satisfies the second-order $P D^{\alpha}$-type ILC algorithm and the initial state of each iteration satisfies (18), then there exists positive $p$ such that $K_{1}+K_{2}<1$, and $K_{3} \longrightarrow 0$. Since $k \longrightarrow \infty,\left\|\Delta u_{k+1}\right\|_{L^{p}}$ is uniformly bounded, which guarantees that $\lim _{k \rightarrow \infty}\left\|e_{k}\right\|_{\lambda}=0$ and $t \in J$.

Proof. According to Lemma 1, we yield

$$
\begin{aligned}
z_{k+1}(t)= & t^{\alpha-1} \mathscr{E}_{\alpha, \alpha}(A, t) z_{0}+\int_{0}^{t}(t-s)^{\alpha-1} E_{\alpha, \alpha}\left(A(t-s)^{\alpha}\right) \\
& \cdot\left(B u_{k+1}(s)+\omega(t)\right) \mathrm{d} s, \\
\Delta z_{k+1}(t)= & \int_{0}^{t}(t-s)^{\alpha-1} E_{\alpha, \alpha}\left(A(t-s)^{\alpha}\right) B\left(\Delta u_{k+1}(s)+\omega(t)\right) \mathrm{d} s, \\
\Delta z_{k}(t)= & \int_{0}^{t}(t-s)^{\alpha-1} E_{\alpha, \alpha}\left(A(t-s)^{\alpha}\right) B\left(\Delta u_{k}(s)+\omega(t)\right) \mathrm{d} s,
\end{aligned}
$$

and then,

$$
\begin{aligned}
\Delta u_{k+1}(t)= & r_{1}\left[\Delta u_{k}(t)+\gamma_{1} C \Delta z_{k}(t)+\gamma_{1} D \Delta u_{k}(t)\right. \\
& \left.+\gamma_{2} C\left(A \Delta z_{k}(t)+B \Delta u_{k}(t)\right)\right] \\
& +r_{2}\left[\Delta u_{k-1}(t)+\gamma_{3} C \Delta z_{k-1}(t)+\gamma_{3} D \Delta u_{k-1}(t)\right. \\
& \left.+\gamma_{4} C\left(A \Delta z_{k-1}(t)+B \Delta u_{k-1}(t)\right)\right] \\
= & \left(r_{1}+r_{1} \gamma_{1} D+r_{1} \gamma_{2} C B\right) \Delta u_{k}(t) \\
& +\left(r_{2}+r_{2} \gamma_{3} D+r_{2} \gamma_{4} C B\right) \Delta u_{k-1}(t) \\
& +\left(r_{1} \gamma_{1} C+r_{1} \gamma_{2} C A\right) \int_{0}^{t}(t-s)^{\alpha-1} E_{\alpha, \alpha} \\
& \cdot\left(A(t-s)^{\alpha}\right) B\left(\Delta u_{k}(s)+\omega(t)\right) \mathrm{d} s \\
& +\left(r_{2} \gamma_{3} C+r_{2} \gamma_{4} C A\right) \int_{0}^{t}(t-s)^{\alpha-1} E_{\alpha, \alpha} \\
& \cdot\left(A(t-s)^{\alpha}\right) B\left(\Delta u_{k-1}(s)+\omega(t)\right) \mathrm{d} s .
\end{aligned}
$$

By taking the $L_{p}$ norm, it yields

$$
\begin{aligned}
& \left\|\Delta u_{k+1}\right\|_{L^{p}} \leq\left\|r_{1}+r_{1} \gamma_{1} D+r_{1} \gamma_{2} C B\right\|\left\|\Delta u_{k}(t)\right\|_{L^{p}}+\left\|r_{1} \gamma_{1} C+r_{1} \gamma_{2} C A\right\| \frac{b^{\alpha-(1 / p)} C_{0} M\|B\|}{\sqrt[q]{q(\alpha-1)+1}}\left\|\Delta u_{k}(t)\right\|_{L^{p}} \\
& +\left\|r_{2}+r_{2} \gamma_{3} D+r_{2} \gamma_{4} C B\right\|\left\|\Delta u_{k-1}(t)\right\|_{L^{p}}+\left\|r_{2} \gamma_{3} C+r_{2} \gamma_{4} C A\right\| \frac{b^{\alpha-(1 / p)} C_{0} M\|B\|}{\sqrt[q]{q(\alpha-1)+1}}\left\|\Delta u_{k-1}(t)\right\|_{L^{p}} \\
& +\left\|r_{1} \gamma_{1} C+r_{1} \gamma_{2} C A\right\| \frac{b^{\alpha-(1 / p)} C_{0} M}{\sqrt[q]{q(\alpha-1)+1}}\|\omega\|_{L_{p}}+\left\|r_{2} \gamma_{3} C+r_{2} \gamma_{4} C A\right\| \frac{b^{\alpha-(1 / p)} C_{0} M}{\sqrt[q]{q(\alpha-1)+1}}\|\omega\|_{L_{p}} .
\end{aligned}
$$




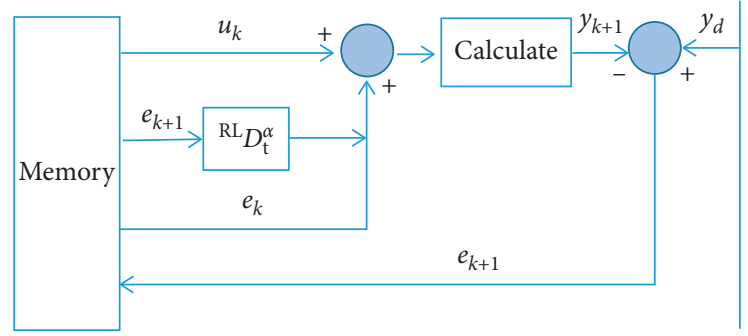

Figure 1: Block diagram of the open- and closed-loop $P D^{\alpha}$-type ILC algorithm.

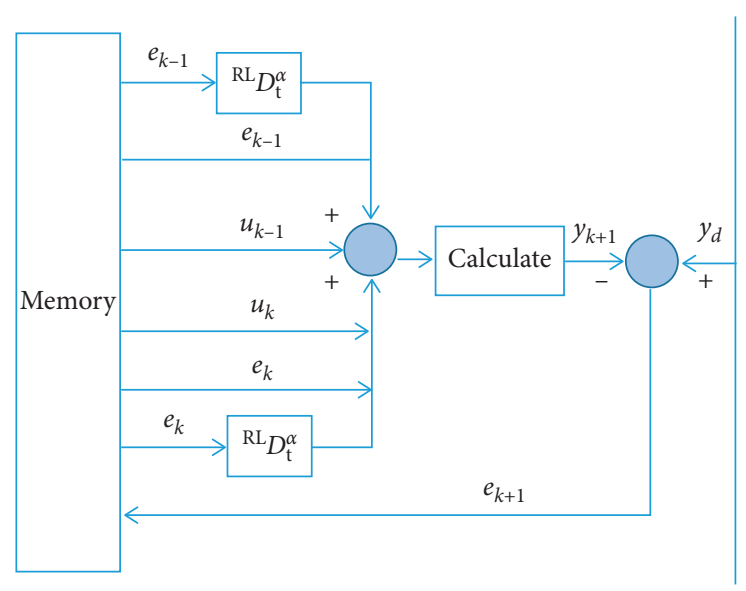

Figure 2: Block diagram of the second-order $P D^{\alpha}$-type ILC algorithm.

For brevity, note that

$$
\begin{aligned}
& K_{1}=\left\|r_{1}+r_{1} \gamma_{1} D+r_{1} \gamma_{2} C B\right\|+\left\|r_{1} \gamma_{1} C+r_{1} \gamma_{2} C A\right\| \frac{b^{\alpha-(1 / p)} C_{0} M\|B\|}{\sqrt[q]{q(\alpha-1)+1}}, \\
& K_{2}=\left\|r_{2}+r_{2} \gamma_{3} D+r_{2} \gamma_{4} C B\right\|+\left\|r_{2} \gamma_{3} C+r_{2} \gamma_{4} C A\right\| \frac{b^{\alpha-(1 / p)} C_{0} M\|B\|}{\sqrt[q]{q(\alpha-1)+1}}, \\
& K_{3}=\left(\left\|r_{1} \gamma_{1} C+r_{1} \gamma_{2} C A\right\|+\left\|r_{2} \gamma_{3} C+r_{2} \gamma_{4} C A\right\|\right) \frac{b^{\alpha-(1 / p)} C_{0} M}{\sqrt[q]{q(\alpha-1)+1}}\|\omega\|_{L_{p}},
\end{aligned}
$$

and one can deduce $\left\|\Delta u_{k+1}\right\|_{L^{p}} \leq K_{1}\left\|\Delta u_{k}\right\|_{L^{p}}+$ $K_{2}\left\|\Delta u_{k-1}\right\|_{L^{p}}+K_{3}$.

There exists a constant $p>0$, which satisfies $K_{1}+K_{2}<1$ and $K_{3} \longrightarrow 0$. Since $k \longrightarrow \infty,\left\|\Delta u_{k+1}\right\|_{L^{p}}$ is uniformly bounded. The proof is completed.

\section{Simulations}

In this section, we will give two simulation examples to demonstrate the validity of the algorithms.

5.1. PD ${ }^{\alpha}$-Type ILC with Initial State Error. Consider the following one-dimensional systems as follows:

$$
\left\{{ }^{\mathrm{RL}} D_{t}^{0.6} x(t)=x_{k}^{2}(t)+0.1 u(t)+\omega_{k}(t), \quad t \in J=[1,2], x(0)=2, y(t)=x(t)+0.3 u_{k}(t)+v(t)\right.
$$




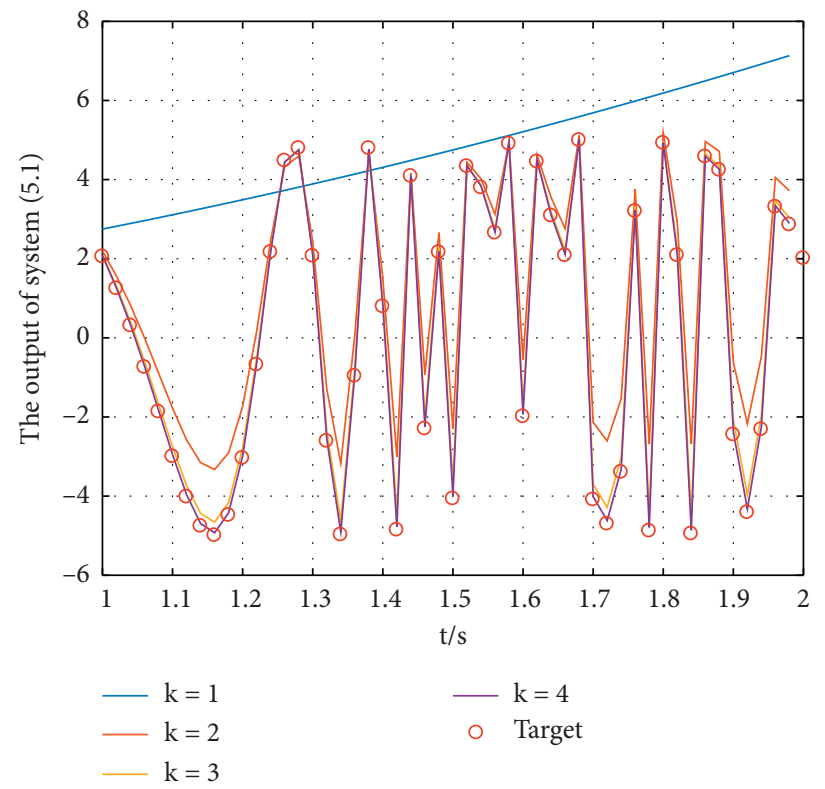

FIGURE 3: Simulation results of output $y_{k}$.

TABLE 1: Numerical simulation of the output of the system in Section 5.1 and the desired trajectory.

\begin{tabular}{|c|c|c|c|c|c|c|c|c|}
\hline$k$ & $y_{k}$ & $y_{d}\left(t_{k}\right)$ & $k$ & $y_{k}$ & $y_{d}\left(t_{k}\right)$ & $k$ & $y_{k}$ & $y_{d}\left(t_{k}\right)$ \\
\hline 1 & 2.0818 & 2.0539 & 18 & -4.9051 & -4.9774 & 35 & 5.0183 & 4.9945 \\
\hline 2 & 1.2580 & 1.2454 & 19 & -0.9237 & -0.9645 & 36 & -4.0133 & -4.0915 \\
\hline 3 & 0.3294 & 0.3088 & 20 & 4.7684 & 4.7910 & 37 & -4.6198 & -4.7037 \\
\hline 4 & -0.7118 & -0.7414 & 21 & 0.8193 & 0.7912 & 38 & -3.3214 & -3.3956 \\
\hline 5 & -1.8284 & -1.8677 & 22 & -4.7823 & -4.8564 & 39 & 3.2206 & 3.1983 \\
\hline 6 & -2.9501 & -2.9989 & 23 & 4.1016 & 4.0857 & 40 & -4.7914 & -4.8791 \\
\hline 7 & -3.9613 & -4.0190 & 24 & -2.2443 & -2.2993 & 41 & 4.9318 & 4.9216 \\
\hline 8 & -4.6935 & -4.7576 & 25 & 2.1803 & 2.1603 & 42 & 2.1176 & 2.0840 \\
\hline 9 & -4.9269 & -4.9936 & 26 & -3.9964 & -4.0669 & 43 & -4.8627 & -4.9535 \\
\hline 10 & -4.4206 & -4.4837 & 27 & 4.3542 & 4.3339 & 44 & 4.5943 & 4.5790 \\
\hline 11 & -2.9888 & -3.0410 & 28 & 3.8435 & 3.7982 & 45 & 4.2592 & 4.2403 \\
\hline 12 & -0.6484 & -0.6824 & 29 & 2.6705 & 2.6515 & 46 & -2.3747 & -2.4479 \\
\hline 13 & 2.1738 & 2.1620 & 30 & 4.9469 & 4.9048 & 47 & -4.3241 & -4.4139 \\
\hline 14 & 4.4456 & 4.4755 & 31 & -1.9316 & -1.9891 & 48 & -2.2423 & -2.3162 \\
\hline 15 & 4.7533 & 4.7927 & 32 & 4.4846 & 4.4505 & 49 & 3.3387 & 3.3090 \\
\hline 16 & 2.0844 & 2.0698 & 33 & 3.1067 & 3.0883 & 50 & 2.8945 & 2.8603 \\
\hline 17 & -2.5569 & -2.6095 & 34 & 2.1099 & 2.0826 & & & \\
\hline
\end{tabular}

with the iterative learning control and initial state error

$$
\left\{\begin{array}{l}
u_{k+1}(t)=u_{k}(t)+0.5 e_{k}(t)+0.5 e_{k+1}^{(\alpha)}(t), \\
x_{k+1}(0)=x_{k}(0)+0.1 e_{k}(t),
\end{array}\right.
$$

where $A x(\cdot)=x(\cdot)^{2}$. Now, we can choose $\alpha=0.6$, $B=0.1, \quad C=1, \quad p=2, \quad \gamma_{1}=\gamma_{2}=0.5$, $\omega(t)=10^{-3} \sin (0.001 t)$, and $\nu(t)=10^{-5}(t)$. For the system, we use the $P D^{\alpha}$-type ILC algorithm and set the initial control $u_{0}(\cdot)=0, y_{d}(t)=5 \sin \left(e^{t^{2}}\right)$, and $t \in(0,2)$. One can calculate $M \approx 3>0, \kappa_{1}=0.47, \kappa_{2}=0.17$, and $\kappa_{3}<0.01=m$, and then, all conditions of Theorem 1 are satisfied.

The state trajectories of system (24) with initial conditions are given in Figure 3 and Table 1, and with the increase of the number of iterations, it can track the desired trajectory gradually. Consistent with the theoretical analysis in the previous section, the algorithm has a faster convergence speed. At the end of the fourth iteration, the algorithm has converged. From Figures 3 and 4, the curve is basically completely fitted, showing that the system algorithm is well robust.

5.2. $P D^{\alpha}$-Type ILC with Random Disturbance. Consider a two-dimensional ILC system; we set $\alpha=0.7, \omega(t)=10^{-10}$ $\sin ((\pi t) / 1000), v(t)=10^{-3}, \quad A\left(\begin{array}{l}x_{1} \\ x_{2}\end{array}\right)=\left(\begin{array}{c}2 x_{1}^{2} \\ x_{2}^{2}\end{array}\right), B=$ $\left(\begin{array}{ll}0 & 1 \\ 1 & 1\end{array}\right), C=\left(\begin{array}{ll}1 & 1 \\ 0 & 1\end{array}\right)$, and $D=\left(\begin{array}{ll}0 & 1 \\ 1 & 1\end{array}\right)$ and construct the second-order $P D^{\alpha}$-type ILC algorithm as follows: 


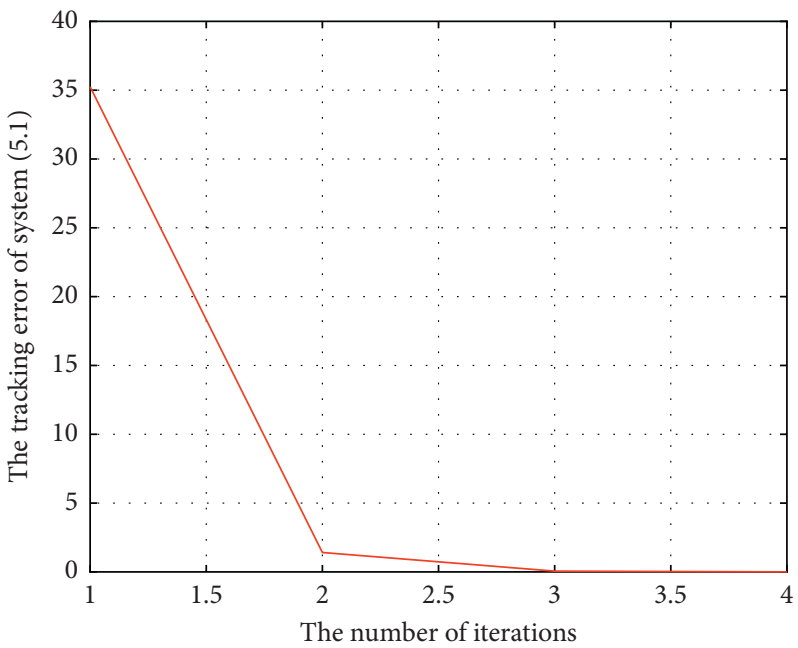

FIgURE 4: The tracking error of the systems.

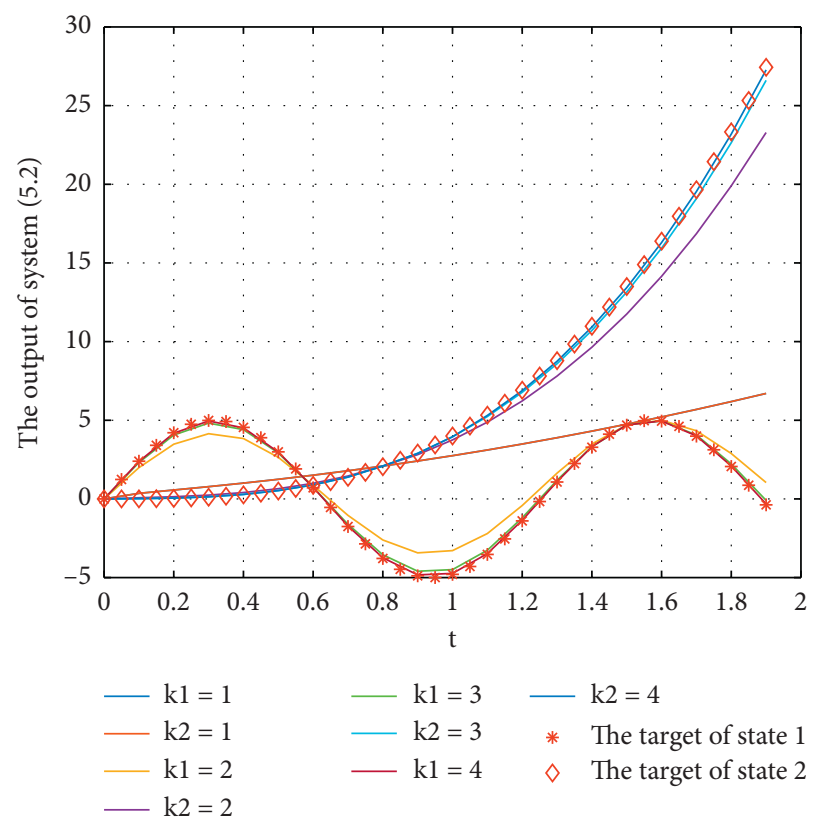

Figure 5: Simulation results of output $y_{k}$.

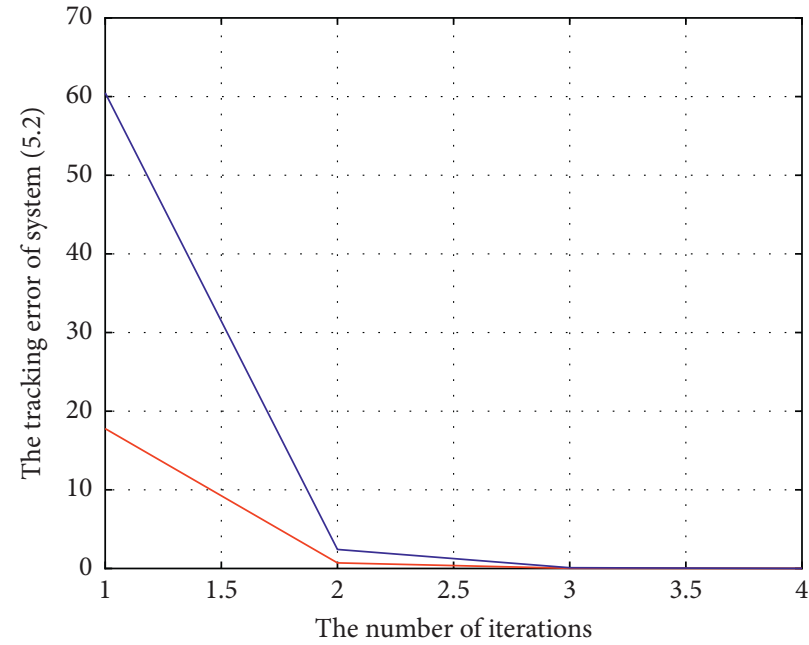

FIgURE 6: The tracking error of the system. 
Table 2: Numerical simulation of the output of the system in Section 5.2 and the desired trajectory.

\begin{tabular}{|c|c|c|c|c|c|}
\hline$k$ & $y_{k}$ & $y_{d}\left(t_{k}\right)$ & $k$ & $y_{k}$ & $y_{d}\left(t_{k}\right)$ \\
\hline 1 & 0 & 0 & 1 & 0 & 0 \\
\hline 2 & 2.3808 & 2.3971 & 2 & 0.0181 & 0.0040 \\
\hline 3 & 4.1782 & 4.2073 & 3 & 0.0534 & 0.0320 \\
\hline 4 & 4.9538 & 4.9874 & 4 & 0.1349 & 0.1080 \\
\hline 5 & 4.5182 & 4.5464 & 5 & 0.2860 & 0.2560 \\
\hline 6 & 2.9784 & 2.9923 & 6 & 0.5299 & 0.5000 \\
\hline 7 & 0.7378 & 0.7056 & 7 & 0.8898 & 0.8640 \\
\hline 8 & -1.7256 & -1.7539 & 8 & 1.3887 & 1.3720 \\
\hline 9 & -3.7370 & -3.7840 & 9 & 2.0900 & 2.0480 \\
\hline 10 & -4.8293 & -4.8876 & 10 & 2.8958 & 2.9160 \\
\hline 11 & -4.7343 & -4.7946 & 11 & 3.9500 & 4.0000 \\
\hline 12 & -3.4746 & -3.5277 & 12 & 5.3063 & 5.3240 \\
\hline 13 & -1.3580 & -1.3970 & 13 & 6.8846 & 6.9120 \\
\hline 14 & 1.0981 & 1.0755 & 14 & 8.7488 & 8.7880 \\
\hline 15 & 3.3259 & 3.2849 & 15 & 10.9227 & 10.9760 \\
\hline 16 & 4.7019 & 4.6899 & 16 & 13.4300 & 13.5000 \\
\hline 17 & 4.9573 & 4.9467 & 17 & 16.2946 & 16.3840 \\
\hline 18 & 4.0060 & 3.9924 & 18 & 19.5403 & 19.6520 \\
\hline 19 & 2.0936 & 2.0605 & 19 & 23.1909 & 23.3280 \\
\hline 20 & -0.3191 & -0.3757 & 20 & 27.2702 & 27.4360 \\
\hline
\end{tabular}

$$
\begin{aligned}
u_{k+1}(t)= & 0.1\left[u_{k}(t)+0.1 e_{k}(t)+0.1 e_{k}^{(\alpha)}(t)\right] \\
& +0.1\left[u_{k-1}(t)+0.2 e_{k-1}(t)+0.2 e_{k-1}^{(\alpha)}(t)\right] \\
k & =2,3, \ldots
\end{aligned}
$$

We also select other parameters and initial values of the algorithm as follows: $\quad u_{0}(\cdot)=0, \quad y_{d}(t)=\left(\begin{array}{l}y_{1 d}(t) \\ y_{2 d}(t)\end{array}\right)$ $=\left(\begin{array}{c}5 \sin (t) \\ 4 t^{3}\end{array}\right), t \in(0,1.9), \quad r_{1}=1, r_{2}=0.5, \gamma_{1}=1$, and $\gamma_{2}$ $=0.5$. It is easy to show that $M \approx 3>0, K_{1}=0.264, K_{2}=0.428$, and $K_{3} \longrightarrow 0$, and all conditions of Theorem 2 are satisfied. In the simulation, $* * *$ denotes the desired trajectory of state $1, \diamond \diamond \diamond$ denotes the desired trajectory of state 2 , and solid lines (--) in different colors denote the output of the system. In Figure 5, we use $\mathrm{k} 1$ to represent the iteration of state 1 and use $\mathrm{k} 2$ to represent the iteration of state 2 , and the tracking error is shown in Figure 6, which implies the number of iterations and tracking error.

From Figure 6 and Table 2, one can find that the tracking error tends to zero quickly, so the output of the system can track the desired trajectory almost perfectly.

\section{Conclusion}

In this paper, we show the concept of mild solutions of the $\mathrm{R}-\mathrm{L}$ fractional system and considered two cases of the $P D^{\alpha}$-type ILC algorithm. The sufficient conditions of robustness analysis of the $P D^{\alpha}$-type ILC algorithm with uncertain disturbances were given by the corresponding theorems and proved. At last, two R-L fractional examples are given to demonstrate the results.

\section{Data Availability}

The data used to support the findings of this study are included within the article.

\section{Conflicts of Interest}

The authors declare that they have no conflicts of interest regarding the publication of this paper.

\section{Authors' Contributions}

The authors contributed equally to this work, and all authors read and approved the final manuscript.

\section{Acknowledgments}

This work was supported by the NSF of China (no. 11661084) and Guizhou Province Department of Education Fund ([2016]046, Qian Jiao He KY[2020]093, and Qian Ke He Ping Tai Ren Cai [2018]5784-08).

\section{References}

[1] X. Liu, J. Wang, and D. O'Regan, “On the approximate controllability for fractional evolution inclusions of sobolev and clarke subdifferential type," IMA Journal of Mathematical Control and Information, vol. 36, no. 1, pp. 1-17, 2019.

[2] Z. Bien and J. X. Xu, Iterative Learning Control Analysis: Design, Integration and Applications, Springer, Berlin, Germany, 1998.

[3] Y. Q. Chen and C. Wen, Iterative Learning Control: Convergence, Robustness and Applications, Springer-Verlag, Berlin, Germany, 1999.

[4] M. Norrlof, "Iterative learning control: Analysis, Design, and Experiments, Linkoping Studies in Science and Technology," Dissertations, Linköping University, Linköping, Sweden, 2000. 
[5] J. X. Xu and Y. Tan, Linear and Nonlinear Iterative Learning Control, Spring-Verlag, Berlin, Germany, 2003.

[6] Y. Wang, F. Gao, and F. J. Doyle III, "Survey on iterative learning control, repetitive control, and run-to-run control," Journal of Process Control, vol. 19, no. 10, pp. 1589-1600, 2009.

[7] J. V. Wijdeven, T. Donkers, and O. Bosgra, "Iterative Learning Control for uncertain systems: robust monotonic convergence analysis," Automatica, vol. 45, no. 10, pp. 2383-2391, 2009.

[8] J. X. Xu, "A survey on iterative learning control for nonlinear systems," International Journal of Control, vol. 84, no. 3, pp. 1275-1294, 2011.

[9] Y. Li, Y. Q. Chen, and H. S. Ahn, "Fractional-order iterative learning control for fractional-order systems," Asian Journal of Control, vol. 13, no. 1, pp. 1-10, 2011.

[10] Y.-H. Lan, "Iterative learning control with initial state learning for fractional order nonlinear systems," Computers \& Mathematics with Applications, vol. 64, no. 10, pp. 3210-3216, 2012.

[11] X. Liu, Y. Li, and Y. Liu, "The convergence of iterative learning control for some fractional system," Advances in Difference Equations, vol. 132, pp. 1-12, 2017.

[12] M.-T. Lin, C.-L. Yen, M.-S. Tsai, and H.-T. Yau, "Application of robust iterative learning algorithm in motion control system," Mechatronics, vol. 23, no. 5, pp. 530-540, 2013.

[13] D. A. Haghighi and S. Mobayen, "Design of an adaptive supertwisting decoupled terminal sliding mode control scheme for a class of fourth-order systems," ISA Transactions, vol. 75, pp. 216-225, 2018.

[14] S. Mobayen, "Chaos synchronization of uncertain chaotic systems using composite nonlinear feedback based integral sliding mode control," ISA Transactions, vol. 77, pp. 100-111, 2018.

[15] S. Mobayen, "A novel global sliding mode control based on exponential reaching law for a class of underactuated systems with external disturbances," Journal of Computational and Nonlinear Dynamics, vol. 11, Article ID 021011, 9 pages, 2016.

[16] S. Mobayen, "Design of LMI-based sliding mode controller with an exponential policy for a class of underactuated systems," Complexity, vol. 21, no. 5, pp. 117-124, 2014.

[17] Y. Li and W. Jiang, "Fractional order nonlinear systems with delay in iterative learning control," Applied Mathematics and Computation, vol. 257, no. 15, pp. 546-552, 2015.

[18] T. Lan and H. Lin, "Accelerated modify approach for initial state error iterative learning control in sense of Lebesgue-p norm," Control and Decision, vol. 31, no. 3, pp. 429-434, 2016.

[19] K. Zhang and G. Peng, "Convergence analysis of PD ${ }^{03 b 1}$-type fractional order iterative learning control in the sense of $L_{p}$ norm," Systems Engineering and Electronics, vol. 39, no. 10, pp. 2285-2290, 2017.

[20] K. S. Miller and B. Ross, An Introduction to the Fractional Calculus and Differential Equations, John Wiley, Hoboken, NJ, USA, 1993.

[21] A. A. Kilbas, H. M. Srivastava, and J. J. Trujillo, "Theory and applications of fractional differential equations," in NorthHolland Mathematics StudiesElsevier Science B.V., Amsterdam, The Netherlands, 2006.

[22] V. Lakshmikantham, S. Leela, and J. Vasundhara Devi, Theory of Fractional Dynamic Systems, Cambridge Academic Publishers, Cambridge, UK, 2009.

[23] K. Diethelm, The Analysis of Fractional Differential Equations, Springer-Verlag, Berlin, Germany, 2010.

[24] G. M. Mophou and G. M. N'Guérékata, "Existence of mild solutions of some semilinear neutral fractional functional evolution equations with infinite delay," Applied Mathematics and Computation, vol. 216, no. 1, pp. 61-69, 2010.

[25] X. Liu, Z. Liu, and M. Bin, "Approximate controllability of impulsive fractional neutral evolution equations with Riemann-Liouville fractional derivatives," Journal of Computational Analysis and Applications, vol. 17, no. 3, pp. 468-485, 2014.

[26] W. Jiang, "The controllability of fractional control systems with control delay," Computers \& Mathematics with Applications, vol. 64, no. 10, pp. 3153-3159, 2012.

[27] E. Bazhlekova, Fractional Evolution Equations in Banach Spaces, Ph.D Thesis, Eindhoven University of Technology, Eindhoven, Netherlands, 2001. 